\title{
Analytic inclusion of the scale dependence of the anomalous dimension matrix in Standard Model Effective Theory
}

\author{
Andrzej J. Buras ${ }^{a, b}$ and Martin Jung ${ }^{c}$ \\ ${ }^{a}$ TUM Institute for Advanced Study, \\ Lichtenbergstr. 2a, D-85748 Garching, Germany \\ ${ }^{b}$ Physik Department, Technische Universität München, \\ James-Franck-Straße, D-85748 Garching, Germany \\ ${ }^{c}$ Excellence Cluster Universe, Technische Universität München, \\ Boltzmannstr. 2, D-85748 Garching, Germany \\ E-mail: andrzej.buras@tum.de, martin.jung@tum.de
}

ABSTRACT: The renormalization group equations (RGEs) in Standard Model effective theory are usually either solved analytically, neglecting the scale dependence of gauge and Yukawa couplings, or numerically without such approximations. We present analytic solutions of RGEs that take into account the dominant scale dependence of the anomalousdimension matrix due to the running of the QCD coupling $\alpha_{s}$ and of the top-Yukawa coupling. We consider first the case for which a given operator is generated directly through mixing with the parent operator whose Wilson coefficient is non-vanishing at the new physics scale. Subsequently we consider the case of two-step running, in which two operators do not mix directly, but only via a third mediator operator. We generalize these solutions to an arbitrary number of operators and show how even in this case analytic solutions can be obtained.

Keywords: Beyond Standard Model, Effective Field Theories, Renormalization Group, Resummation

ARXIV EPRINT: 1804.05852 


\section{Contents}

1 Introduction 1

2 Basic setup 3

3 Scale-independent ADM 5

3.1 One step running 5

3.2 Two-step running 6

4 Analytical solution of RGEs $\quad 6$

4.1 The case of a single Wilson coefficient 6

$\begin{array}{llr}4.2 & \text { General case of one step running } & 9\end{array}$

$\begin{array}{lll}4.3 \text { General case of two step running } & 10\end{array}$

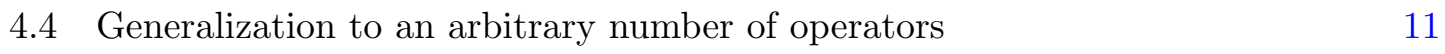

$\begin{array}{ll}4.5 & \text { Special cases of weak mixing } \\ \end{array}$

$\begin{array}{llr}5 & \text { Numerical analysis } & 14\end{array}$

$\begin{array}{lll}6 & \text { Summary } & 15\end{array}$

A General two-step running with an arbitrary number of operators $\quad 16$

\section{Introduction}

The absence of any signal in direct searches for particles beyond the Standard Model (SM) at the LHC renders a scenario likely in which the scale $\Lambda$ of such potential particles is much larger than the electroweak scale. The resulting hierarchy can be used together with the assumption of linearly realized electroweak symmetry breaking to formulate the so-called Standard Model Effective Theory (SMEFT), provided no undiscovered weaklycoupled light particles exist, like axions or sterile neutrinos. This framework incorporates the full SM gauge symmetry which is unbroken at the high scale $\Lambda$ and allows to investigate its implications on observables measured at scales significantly below $\Lambda$. Technically this is realized via an operator product expansion, where all the SM degrees of freedom are kept as dynamical degrees of freedom and only the NP is integrated out, yielding dimension-six operators built out of SM fields that are invariant under the full SM gauge symmetry. This type of effective theory is usually called SMEFT, because it reduces at low energies to the SM. Yet, the presence of dimension-six operators whose Wilson coefficients can in principle be arbitrary can introduce very significant modifications to the SM phenomenology. 
The effective Lagrangian of SMEFT at dimension six is given as follows:

$$
\mathcal{L}^{(6)}=\sum_{k} C_{k}^{(6)} Q_{k}^{(6)}
$$

with the contributing operators classified in full generality in $[1,2]$, the latter article for the first time providing an irreducible basis which is now standard. The corresponding renormalization group analysis at leading order of all these operators has been carried out in $[3-5] .{ }^{1}$ The renormalization group equations (RGEs) in SMEFT derived there often involve many operators mixing with each other. The corresponding anomalous dimension matrix (ADM) depends not only on the three gauge couplings of the SM, but also on fermion Yukawa couplings, in particular the top-quark Yukawa coupling, and quartic Higgs couplings.

Given this complicated structure, solutions to RGEs in SMEFT are typically obtained in one of two ways: either analytically, assuming the ADM to be constant and furthermore commonly considering only the first leading logarithm, or numerically as in [6-8], taking the scale-dependence of the ADM into account, but making further analytical insight difficult. Although the former procedure can be useful in showing the overall importance of renormalization group effects, see for instance [9, 11-16], we prefer to avoid the assumption of a scale-independent ADM, since the anomalous dimensions involved in fact typically show a pronounced scale dependence. However, analytical solutions are very useful, since they are easy to use and facilitate applying constraints at different scales. Moreover, they give some insight into the pattern of the dominant dynamical effects. The main goal of our paper is therefore to provide analytic solutions for the relevant RGEs, including the phenomenlogically relevant effects from the scale dependence of the ADM, specifically the running of the strong and relevant Yukawa couplings. In order to achieve this and to resum the leading logarithms to all orders in perturbation theory, we exploit a distinct hierarchy in the different running effects:

1. The running of the strong and top-Yukawa couplings themselves is dominated by SM physics; while effects from NP are possible, they can be safely assumed to be at most of the order of the neglected NLO contributions.

2. For operators present at the scale $\Lambda$, the RG effects due to the latter two couplings are dominant, since they are the only $\mathcal{O}(1)$ couplings.

3. The scale-dependence of $g_{1}$ and $g_{2}$ is much weaker than that of $g_{3}$ (or equivalently $\alpha_{s}$ ) and $y_{t}$; we hence consider $g_{1,2}$ fixed to their values at the electroweak scale.

We therefore solve the RGEs in a step-wise fashion: we first discuss the scale dependence of the strong and top Yukawa couplings. We use these results to solve the self-mixing of the parent operators generated at the NP scale $\Lambda$. Finally, taking the latter solution into account, we consider the running of the child operators generated via weak mixing, including their self-mixing.

\footnotetext{
${ }^{1}$ See http://einstein.ucsd.edu/smeft/ for errata.
} 
Solving the RGEs including the running of both $\alpha_{s}$ and $y_{t}$ is not trivial, even with this hierarchy of different effects; while we discuss the exact inclusion of both couplings, we achieve a much simpler form due to the crucial observation that

$$
\frac{y_{t}^{2}(\mu)}{\alpha_{s}(\mu)} \approx \text { const. }
$$

The weak $\mu$-dependence of the right-hand side at leading order in $\alpha_{s}$ is discussed below and shown to have a negligible effect, such that both effects can be included using a simple, very accurate approximation. Higher order corrections are unimportant at scales $\mu \geq \mu_{\mathrm{EW}}$ considered by us. They would become more important at $\mu \ll \mu_{\mathrm{EW}}$, but $y_{t}(\mu)$ does not run at these scales as the top quark is integrated out at $\mu \approx \mu_{\mathrm{EW}}$. Nevertheless, the relation still holds approximately for the remaining Yukawa couplings.

Our paper is organized as follows: in section 2 we recall the relevant formulae for RGEs and specify our assumptions. In section 3 we recall for pedagogical reasons the solutions of RGEs when the ADM is assumed to be scale independent. Here we consider also the interesting case mentioned in the abstract when the coefficient of the first leading logarithm vanishes so that the solution involves the square of $\ln (\Lambda / \mu)$. In section 4 , the main section of our paper, we derive the analytic solutions of RGEs under the assumptions listed above. We demonstrate our method first for the case of a single non-vanishing Wilson coefficient at $\mu=\Lambda$ and generalise it systematically to an arbitrary number of operators. The examples given there demonstrate the precision of our analytical formulae. Our brief numerical analysis in section 5 demonstrates the application of the developed framework very explicitly, in order to facilitate its application. In section 6 we summarize our results by presenting table 1, which guides the reader to the main results of our paper and should simplify their usage.

\section{Basic setup}

The one-loop RGE can generically be written as

$$
\dot{\mathbf{C}} \equiv 16 \pi^{2} \frac{d \mathbf{C}}{d \ln \mu}=\hat{\gamma}(\mu) \mathbf{C},
$$

where $\mathbf{C}=\left(C_{1}, C_{2}, C_{3}, \ldots\right)^{T}$ contains the Wilson coefficients of contributing operators and $\hat{\gamma}$ is a general, scale-dependent anomalous dimension matrix that depends on gauge and Yukawa couplings. Note that we do not use the traditional notation with the transpose of the anomalous dimension matrix in order to agree with [3-5], where anomalous dimensions are defined for Wilson coefficients and not operators. In our examples we will mostly use the conventions of these papers specifying exceptions.

We first consider a typical scenario in which NP generates a subset of parent operators $\mathcal{O}_{i}$ of the SMEFT at some high scale $\Lambda \gg \mu_{\mathrm{EW}}$. The RG-running of this subset down to $\mu_{\mathrm{EW}}$, described by the RGEs in eq. (2.1), has the following implications:

- Creation of non-vanishing coefficients of the child operators $\mathcal{O}_{k}$. In order to distinguish these operators from parent operators $\mathcal{O}_{i}$, we use the indices $(k, l)$ for the former, while $(i, j)$ are used for the latter. 
- Mixing between the parent operators $\mathcal{O}_{i}$ (including their self-mixing), modifying their coefficients.

- Mixing between the child operators $\mathcal{O}_{k}$ (including their self-mixing), modifying their coefficients.

- Mixing of the child operators back into the parent operators.

Considering electroweak, strong and Yukawa interactions, the RG effects show additional hierarchies:

- Strong mixing due to the QCD and (top) Yukawa couplings can generate large effects, and exhibits a strong scale dependence. Note that these two contributions are qualitatively different: while Yukawa interactions can provide chiral flips, this is not possible for QCD. We define parent operators to include all operators with $C_{i}(\Lambda) \neq 0$, together with those generated from the $\mathcal{O}_{i}$ via strong mixing.

- The child operators $\mathcal{O}_{k}$ are hence by definition generated via weak mixing. The corresponding anomalous dimensions include electroweak gauge couplings and/or Yukawa couplings from lighter fermions. As such, they fulfill generically $\gamma_{\text {weak }} / \gamma_{\text {strong }} \ll 1$. Analogously to parent operators, the class of child operators includes also those not directly generated via weak mixing, but via strong mixing from operators generated via weak mixing. As already stated in the introduction, the scale dependence of $g_{1,2}$ will be neglected.

- Since by definition $C_{k}(\Lambda) \equiv 0$, the mixing of child into parent operators is of higher order, even without additional hierarchies. Given the definitions above, it is actually at least suppressed as $\gamma_{\text {weak }}^{2} / \gamma_{\text {strong relative to strong-mixing contributions, and hence }}$ negligible.

Schematically, we obtain the following block form for the ADM, writing $P$ and $C$ for parent and child operators, respectively:

$$
\hat{\gamma}=\left(\begin{array}{ll}
\hat{\gamma}_{\text {strong }}^{P} & 0 \\
\hat{\gamma}_{\text {weak }}^{P \rightarrow C} & \hat{\gamma}_{\text {strong }}^{\mathrm{C}}
\end{array}\right), \quad \text { with } \quad \mathbf{C}(\mu)=\left(\begin{array}{l}
\mathbf{C}_{\mathrm{P}}(\mu) \\
\mathbf{C}_{\mathrm{C}}(\mu)
\end{array}\right) \quad \text { and } \quad \mathbf{C}(\Lambda)=\left(\begin{array}{c}
\mathbf{C}_{\mathrm{P}}(\Lambda) \\
0
\end{array}\right) .
$$

Our goal is to include all phenomenologically relevant effects in this setup.

Formally, the solution of eq. (2.1) can be written as

$$
\mathbf{C}(\mu)=\exp \left[\int_{\ln \Lambda}^{\ln \mu} \frac{\hat{\gamma}(\tilde{\mu})}{16 \pi^{2}} d \ln \tilde{\mu}\right] \mathbf{C}(\Lambda),
$$

and the result for $\mathbf{C}(\mu)$ can in principle be obtained by numerically performing the integral in the exponential. In this manner all the effects discussed above can be taken into account. Moreover, as the result is written in terms of an exponential, all leading logarithms are summed up to all orders of perturbation theory. It should be remarked that in the presence of two-loop ADMs (2.3) should be generalized to include $T_{g}$ ordering that takes into account that one-loop and two-loop ADMs generally do not commute with each other. However, in this paper we will confine our discussion to one-loop ADMs. 


\section{Scale-independent ADM}

We begin with the simplest scenario in which $\hat{\gamma}$ is scale independent to find

$$
\mathbf{C}(\mu)=\exp \left[-\frac{\hat{\gamma}}{16 \pi^{2}} \ln \left(\frac{\Lambda}{\mu}\right)\right] \mathbf{C}(\Lambda)
$$

The leading logarithms are summed to all orders, but assuming $\hat{\gamma}$ to be scale independent is an approximation that will be remedied in the next section.

\subsection{One step running}

If the argument of the exponential in eq. (3.1) is sufficiently below unity, we can expand it to obtain

$$
\mathbf{C}(\mu)=\left[\hat{1}-\frac{\hat{\gamma}}{16 \pi^{2}} \ln \left(\frac{\Lambda}{\mu}\right)\right] \mathbf{C}(\Lambda)
$$

with $\hat{1}$ denoting the unit matrix. This result is often encountered in the literature. The effects included in this rough solution can be made explicit by simply performing the multiplication of $\hat{\gamma}$ and $\mathbf{C}(\Lambda)$, keeping our conventions for the indices $(i, j, k)$ in mind: one finds $(\mu<\Lambda)$

$$
C_{k}(\mu)=-\frac{\hat{\gamma}_{k i}}{16 \pi^{2}} \ln \left(\frac{\Lambda}{\mu}\right) C_{i}(\Lambda), \quad C_{j}(\mu)=\left[\delta_{j i}-\frac{\hat{\gamma}_{j i}}{16 \pi^{2}} \ln \left(\frac{\Lambda}{\mu}\right)\right] C_{i}(\Lambda) .
$$

We observe the following:

- The first result above describes the generation of child operators from parent operators due to weak mixing.

- The second one describes the evolution of parent operators due to strong mixing, including self mixing due to $\hat{\gamma}_{i i}$.

- While the self mixing affects the values of the parent coefficients $C_{i}(\mu)$, it does not have impact on child coefficients $C_{k}(\mu)$ at this order.

- Similarly, the strong mixing among the child coefficients has no impact on other child coefficients at this order, even if $\hat{\gamma}_{k l} \neq 0$.

The latter two points can be improved by expanding eq. (3.1) to higher orders (or using the full solution), although effects not taken into account in eq. (3.3) and listed above will generally be subleading. Generally higher powers of $\hat{\gamma}_{\text {weak }}$ will be neglected in this expansion; however, such contributions can be relevant when the contributions at first order are absent or heavily suppressed, e.g. by several Yukawa couplings of light fermions. We will discuss this case now, still without the inclusion of the scale-dependence in $\hat{\gamma}$. 


\section{$3.2 \quad$ Two-step running}

The first formula in eq. (3.3) tells us that for a given element $\hat{\gamma}_{k i}=0$ no mixing occurs between the operators $\mathcal{O}_{k}$ and $\mathcal{O}_{i}$ at one-loop level, and consquently $C_{k}(\mu)=0$ in ordinary perturbation theory. On the one hand, such mixing could take place at two-loop level, in which case the contribution would be of the order $\gamma_{\text {weak }}^{2} \ln (\Lambda / \mu)$. However, it turns out that the renormalization group improved solution (3.1) can generate non-vanishing $C_{k}(\mu)$ through the so-called two-step running, even if $\hat{\gamma}_{k i}=0$ at one-loop. Such contributions receive an additional enhancement by $\ln (\Lambda / \mu)$ compared to the two-loop matching contribution, which can render them dominant for high scales $\Lambda$. We consider only these enhanced contributions in the following. This mechanism was primarily discussed in the context of electric dipole moments $[9,11,12]$, where a situation occurs when a given operator of interest does not mix directly with a second operator that enters an experimental observable, but does so via a third mediator operator. In spite of the presentations in $[9,11,12]$ we describe this case again in an attempt to exhibit the resulting structure more clearly.

To be specific, we consider the following situation: the coefficient of an operator $\mathcal{O}_{i}$ is the only one with a non-zero value at some high scale $\Lambda$. At some much lower scale $\mu$ an observable is determined by the value of a coefficient of an operator $\mathcal{O}_{k}$, which does not mix directly with $\mathcal{O}_{i}$, but $\mathcal{O}_{i}$ mixes into a third operator $\mathcal{O}_{m}$ and $\mathcal{O}_{m}$ mixes into $\mathcal{O}_{k}$. The one-loop RGE and its solution in the approximation of scale-independent $\hat{\gamma}$ are again given by eqs. (2.1) and (3.1), respectively, where additionally $\mathbf{C}=\left(C_{i}, C_{m}, C_{k}\right)^{T}$ and

$$
\mathbf{C}(\Lambda)=\left(C_{i}(\Lambda), 0,0\right)^{T}, \quad \hat{\gamma}_{k i}=0, \hat{\gamma}_{m i} \neq 0 \quad \text { and } \quad \hat{\gamma}_{k m} \neq 0
$$

Expanding the exponential, we find that a non-vanishing result for $C_{k}(\mu)$ is obtained first at second order which introduces a factor $1 / 2$ and a logarithm squared. One can also check that as long as eqs. (3.4) are satisfied, the result for $C_{k}(\mu)$ is independent of other entries in $\hat{\gamma}$. The latter enter first at third order and can be neglected. We thus find the leading contribution

$$
C_{k}(\mu)=\frac{1}{2} \hat{\gamma}_{k m} \hat{\gamma}_{m i}\left[\frac{1}{16 \pi^{2}} \ln \left(\frac{\Lambda}{\mu}\right)\right]^{2} C_{i}(\Lambda)
$$

This result is trivially extended to block-diagonal forms of $\hat{\gamma}$. Needless to say that eq. (3.1) allows for the generalization of this result to an arbitrary number of operators and to contributions via arbitrary levels of mediator operators, which, however, will typically give negligible contributions.

\section{Analytical solution of RGEs}

\subsection{The case of a single Wilson coefficient}

We will next take into account the scale dependence of $\hat{\gamma}$ resulting from $\alpha_{s}(\mu)$ and $y_{q}(\mu)$. For pedagogical reasons we start with the derivations for a single coefficient $\mathcal{C}_{i}$ with the corresponding anomalous dimension $\gamma_{i}(\mu)$ to be explicitly given below. The relevant RG equation remains eq. (2.1) and its formal solution eq. (2.3), both written for a single coefficient. To perform the integration in eq. (2.3), we first reiterate that the only two 


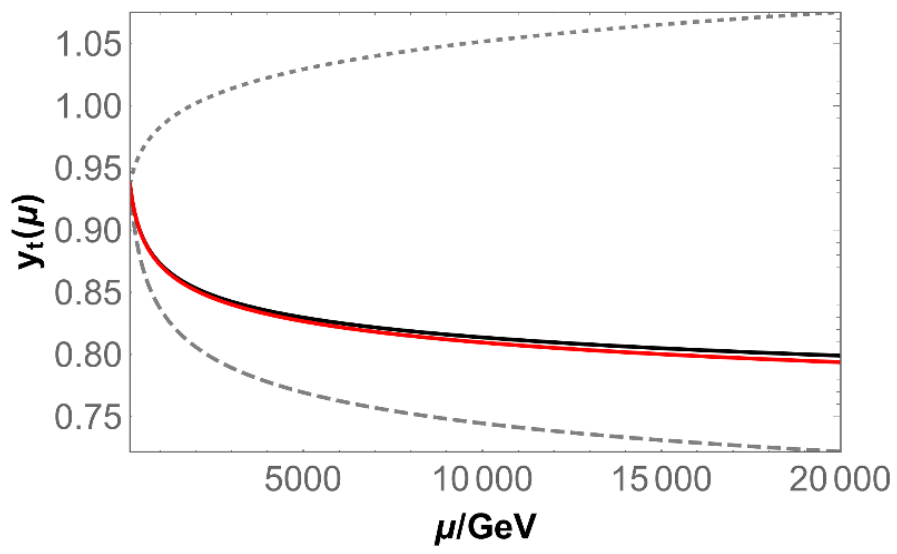

Figure 1. The running of the top Yukawa coupling in different approximations: grey-dashed: only $\alpha_{s}$ running; grey-dotted: only $y_{t}^{3}$ running; black: exact solution [19]; red: our approximation, see eqs. (4.3), (4.4).

numerically important contributions in self mixing are the ones from the strong and the top-Yukawa couplings that can both appear in $\gamma_{i}(\mu)$. Regarding the scale dependence of the strong coupling, we use the standard leading-logarithmic solution

$$
\alpha_{s}(\mu)=\frac{\alpha_{s}\left(\mu_{0}\right)}{1+\alpha_{s}\left(\mu_{0}\right) \frac{\beta_{0}}{2 \pi} \ln \frac{\mu}{\mu_{0}}}
$$

with some reference scale $\mu_{0}$. As far as the top-Yukawa coupling is concerned, we note that its QCD evolution satisfies for $\mu>\mu_{\mathrm{EW}}$ to an excellent approximation

$$
\frac{y_{t}^{2}(\mu)}{\alpha_{s}(\mu)} \approx \text { constant. }
$$

For the top Yukawa, we also take a non-linear term $\sim y_{t}^{3}[17,18]$ approximately into account. Assuming eq. (4.2) to hold for the full solution, we obtain ${ }^{2}$

$$
y_{q}(\mu)=y_{q}\left(\mu_{0}\right)\left[\eta\left(\mu, \mu_{0}\right)\right]^{\epsilon_{y_{q}}}
$$

where we introduced

$$
\eta\left(\mu, \mu_{0}\right) \equiv \frac{\alpha_{s}(\mu)}{\alpha_{s}\left(\mu_{0}\right)}, \quad \epsilon_{y_{q}} \equiv \frac{1}{8 \pi \beta_{0}}\left[4 \pi \gamma_{m}^{(0)}-\frac{9}{2} \frac{y_{q}^{2}\left(\mu_{0}\right)}{\alpha_{s}\left(\mu_{0}\right)}\right],
$$

with $\gamma_{m}^{(0)}=4 C_{F}=8$. The expression for $\epsilon_{y_{q}}$ holds for the Yukawa coupling of any quark, but the non-linear part is only relevant for the top quark. This approximation is excellent over the whole range of considered scales, see figure 1.

Given eq. (4.3), both contributions we consider for the self mixing are proportional to $\alpha_{s}$ to some power. We make these dependencies explicit by writing

$$
\gamma_{i}=h_{\alpha_{s}}^{i} \alpha_{s}+h_{y_{t}}^{i} \alpha_{s}^{2 \epsilon_{y_{t}}},
$$

\footnotetext{
${ }^{2}$ Note that the scale $\mu_{0}$ does not have to be equal to the one in $\alpha_{s}(\mu)$.
} 
such that the quantities $h_{\alpha_{s}}^{i}$ and $h_{y_{t}}^{i}$ are scale-independent, and the lower index differentiates between the two possible terms in this equation. The integration in eq. (2.3) can be simplified by using

$$
d \ln \tilde{\mu}=-16 \pi^{2} \frac{d g_{s}}{\beta_{0} g_{s}^{3}}=-2 \pi \frac{d \alpha_{s}}{\beta_{0} \alpha_{s}^{2}} ;
$$

inserting this into eq. (2.1) and again making the $\alpha_{s^{-}}$-dependence explicit, we obtain

$$
\frac{d \mathcal{C}_{i}}{d \alpha_{s}}=\left(p_{\alpha_{s}}^{i} \alpha_{s}+p_{y_{t}}^{i} \alpha_{s}^{2 \epsilon_{y_{t}}}\right) \alpha_{s}^{-2} \mathcal{C}_{i},
$$

where we introduced

$$
p_{\alpha_{s}}^{i}=-\frac{h_{\alpha_{s}}^{i}}{8 \pi \beta_{0}}, \quad p_{y_{t}}^{i}=-\frac{h_{y_{t}}^{i}}{8 \pi \beta_{0}} .
$$

In this form the integration of eq. (4.7) can be performed easily, yielding

$$
\mathcal{C}_{i}(\mu)=\eta^{p_{\alpha_{s}}^{i} \exp \left[X_{i}(\mu)-X_{i}(\Lambda)\right] \mathcal{C}_{i}(\Lambda)}
$$

where

$$
X_{i}(\mu)=\frac{p_{y_{t}}^{i}}{2 \epsilon_{y_{t}}-1}\left[\alpha_{s}(\mu)\right]^{2 \epsilon_{y_{t}}-1}, \quad \eta=\eta(\mu, \Lambda),
$$

with $\epsilon_{y_{t}}$ defined in eq. (4.4). This expression holds for arbitrary values of $\epsilon_{y_{t}} \neq 1 / 2$. In the limit $\left|2 \epsilon_{y_{t}}-1\right| \ll 1$, we can write

$$
\gamma_{i}=\left(h_{\alpha_{s}}^{i}+h_{y_{t}}^{i} \alpha_{s}^{2 \epsilon_{y_{t}}-1}\right) \alpha_{s} \equiv h_{\mathrm{eff}}^{i} \alpha_{s}
$$

and again neglect the scale dependence in the bracket so that $h_{\mathrm{eff}}^{i}$ is $\mu$-independent. In that case eq. (4.9) simplifies to

$$
\mathcal{C}_{i}(\mu)=\eta^{p_{\text {eff }}^{i}} \mathcal{C}_{i}(\Lambda), \quad p_{\text {eff }}^{i}=-\frac{h_{\text {eff }}^{i}}{8 \pi \beta_{0}}
$$

As an example, we consider top dipole operator $\mathcal{O}_{t B}=\left(\mathcal{O}_{u B}\right)_{33}$ : using the results from refs. $[4,5]$ and eq. (4.3), we obtain

$$
h_{\alpha_{s}}^{i}=\frac{32 \pi}{3} \quad \text { and } \quad h_{y_{t}}^{i}=\frac{15}{2} y_{t}(\Lambda)^{2} \alpha_{s}(\Lambda)^{-2 \epsilon_{y_{t}}} .
$$

The resulting running is shown in figure 2, where we compare the exact solution eq. (4.9) with our approximation in eq. (4.12), the solution for a constant ADM and the solution for the case where the term $\sim y_{t}^{2}$ in the ADM is neglected: while the running due to the top-Yukawa coupling is important to include, we see that in this case the change due to the scale dependence of the ADM is in this case actually negligible. This is due to the fact that the scale dependence of the ADM is in this case a second-order correction: since the coefficient is present at the high scale, already the running due to the scale-independent $\mathrm{ADM}$ is a correction; the relative correction to the effect we are considering is, however, not small, it amounts to $\sim 15 \%$ in this case over the considered range of scales. 


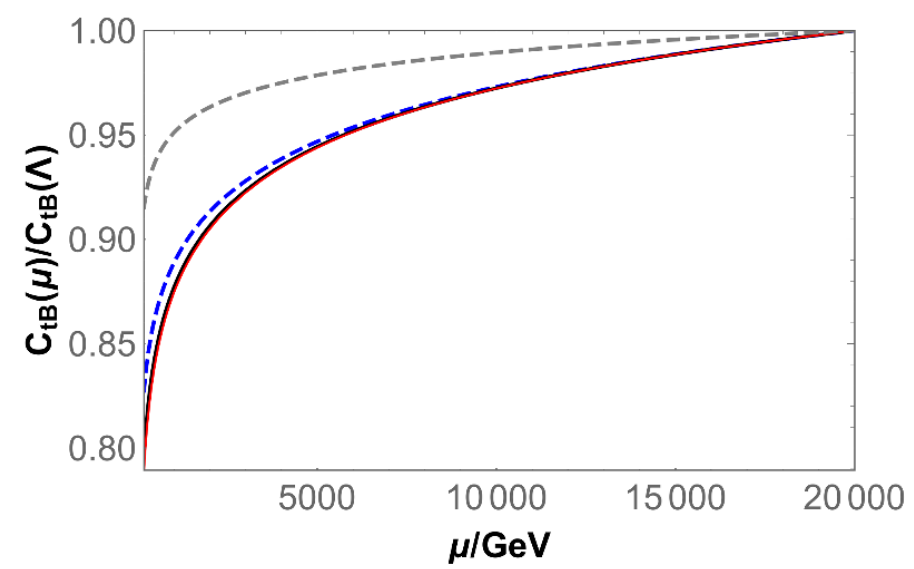

Figure 2. Running of $C_{t B}$ in different scenarios: neglecting the term $\sim y_{t}^{2}$ in the ADM (grey dashed), assuming a scale-independent ADM (blue dashed), exact (black) and with our approximation (red, overlapping with the black line).

\subsection{General case of one step running}

With the solutions in eqs. (4.9) and (4.12) at hand, we return to the RGEs for the operators induced by the ones at the scale $\Lambda$ via weak mixing, with the goal to generalize the expressions in section 3 by including the scale dependence in the anomalous dimensions.

We first consider the case of a single parent operator $\mathcal{O}_{i}$ and a single child operator $\mathcal{O}_{k}$, neglecting the impact of mixing with other operators; the latter effect will be included below. The one-loop RGE is given as follows:

$$
\dot{\mathcal{C}}_{k}=h^{k i} \alpha_{s}^{a_{k i}} \mathcal{C}_{i}+\left(h_{\alpha_{s}}^{k} \alpha_{s}+h_{y_{t}}^{k} \alpha_{s}^{2 \epsilon_{y_{t}}}\right) \mathcal{C}_{k}, \quad \text { with } \quad k \neq i \quad \text { and } \quad \mathcal{C}_{k}(\Lambda)=0,
$$

where again the $\alpha_{s}$-dependence of the anomalous dimensions is made explicit via the arbitrary exponent $a_{k i}$ and constant parameters $h^{k i}$ and $h_{\alpha_{s}, y_{t}}^{k}$. The first term on the right-hand side of this equation describes parent-child mixing, whereas the second term describes the self-mixing of the child operator.

Inserting the result for $\mathcal{C}_{i}(\mu)$ from eq. (4.9) we take the self-mixing of the parent operator into account. The resulting equation can be integrated exactly. We find

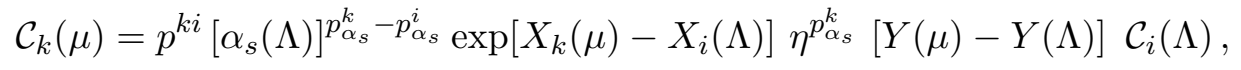

with

$$
\begin{aligned}
p^{k i} & =-\frac{h^{k i}}{8 \pi \beta_{0}} \quad \text { and } \\
Y(\mu) & =\frac{1}{1-2 \epsilon_{y_{t}}}\left(\frac{p_{y_{t}}^{i}-p_{y_{t}}^{k}}{1-2 \epsilon_{y_{t}}}\right)^{\frac{p_{\alpha_{s}}^{i}-p_{\alpha_{s}}^{k}+a_{k i}-1}{1-2 \epsilon_{y_{t}}} \Gamma\left(\frac{1-p_{\alpha_{s}}^{i}+p_{\alpha_{s}}^{k}-a_{k i}}{1-2 \epsilon_{y_{t}}}, \frac{\left[\alpha_{s}(\mu)\right]^{2 \epsilon_{y_{t}}-1}\left(p_{y_{t}}^{i}-p_{y_{t}}^{k}\right)}{1-2 \epsilon_{y_{t}}}\right),}
\end{aligned}
$$

where $\Gamma(s, x)$ denotes the incomplete Gamma function, with the integral representation given as

$$
\int_{u}^{v} d t t^{s-1} e^{-t}=\Gamma(s, v)-\Gamma(s, u) .
$$

All other symbols have been defined previously. 


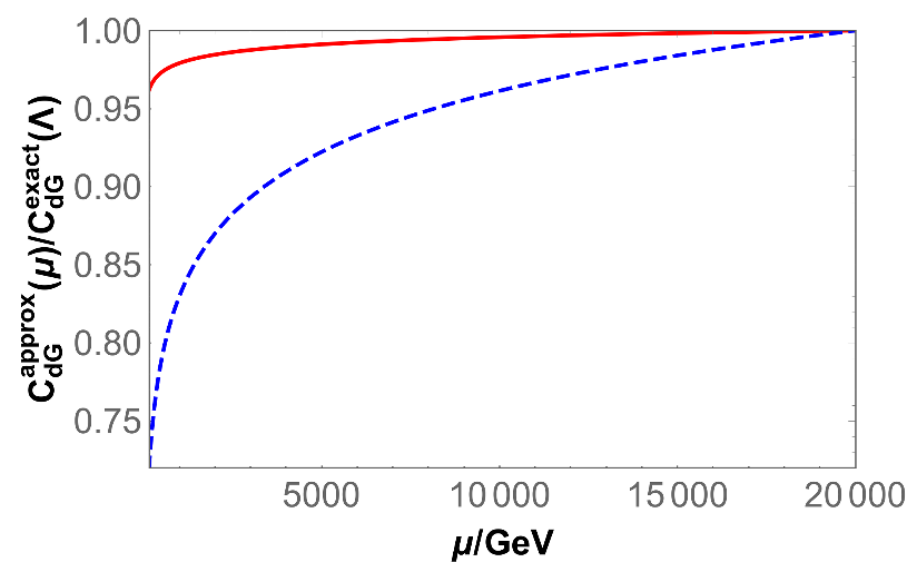

Figure 3. Comparison of the approximations discussed in the text, taking the scale-dependence of the ADM into account (red) or not (blue dashed). Shown is the mixing of $C_{t G}$ into $C_{d G}$ relative to the exact solution.

Inserting instead the approximation eq. (4.12), we obtain the much simpler expression

$$
\mathcal{C}_{k}(\mu)=\frac{p^{k i}}{a_{k i}+p_{\mathrm{eff}}^{i}-p_{\mathrm{eff}}^{k}-1}\left[\alpha_{s}(\Lambda)\right]^{a_{k i}-1}\left(\eta^{a_{k i}+p_{\mathrm{eff}}^{i}-1}-\eta^{p_{\mathrm{eff}}^{k}}\right) \mathcal{C}_{i}(\Lambda),
$$

which again is an excellent approximation. We demonstrate the different approximations in figure 3 , where we consider the one-loop mixing of $\mathcal{O}_{t G}$ into $\mathcal{O}_{d G}$ as an example. As already discussed in the context of self mixing, the influence of the scale dependence is much larger here, since there is no trivial leading-order contribution that is equal in both cases; while the approximation of a scale-independent ADM differs from the exact solution by $\sim 30 \%$ over the considered range, our approximate formula shows less than $4 \%$ deviation.

\subsection{General case of two step running}

We begin with two RG expressions

$$
\begin{aligned}
\dot{\mathcal{C}}_{k}(\mu) & =h^{k m} \alpha_{s}^{a_{k m}} \mathcal{C}_{m}(\mu)+\left(h_{\alpha_{s}}^{k} \alpha_{s}+h_{y_{t}}^{k} \alpha_{s}^{2 \epsilon_{y_{t}}}\right) \mathcal{C}_{k}(\mu), \\
\dot{\mathcal{C}}_{m}(\mu) & =h^{m i} \alpha_{s}^{a_{m i}} \mathcal{C}_{i}(\mu)+\left(h_{\alpha_{s}}^{m} \alpha_{s}+h_{y_{t}}^{m} \alpha_{s}^{2 \epsilon_{y_{t}}}\right) \mathcal{C}_{m}(\mu),
\end{aligned}
$$

together with the solution for $\mathcal{C}_{i}$ obtained previously and with boundary conditions

$$
\mathcal{C}_{k}(\Lambda)=\mathcal{C}_{m}(\Lambda)=0, \quad h^{k i}=0 .
$$

Evidently $\mathcal{O}_{k}, \mathcal{O}_{m}, \mathcal{O}_{i}$ are child, mediator and parent operators, respectively. We solved eq. (4.21) in the last subsection (for $m \rightarrow k$ ), so we can use for $\mathcal{C}_{m}(\mu)$ either the exact expression in eq. (4.15) or the approximate one in eq. (4.19). While the resulting equation still has an exact analytic solution within our setup in the former case, we restrict ourselves for simplicity to the solution using the approximation in eq. (4.19). We hence consider the equation

$$
\frac{d \mathcal{C}_{k}\left(\alpha_{s}\right)}{d \alpha_{s}}=p^{k m} \alpha_{s}^{a_{k m}-2} \mathcal{C}_{m}\left(\alpha_{s}\right)+p_{\text {eff }}^{k} \alpha_{s}^{-1} \mathcal{C}_{k}\left(\alpha_{s}\right)
$$


with $\mathcal{C}_{m}\left(\alpha_{s}\right)$ given in eq. (4.19). The solution reads

$\mathcal{C}_{k}(\mu)=\frac{p^{k m} p^{m i}\left[\alpha_{s}(\Lambda)\right]^{a_{k m}+a_{m i}-2}\left[A_{m i} \eta^{p_{\mathrm{eff}}^{k}}+A_{k m} \eta^{a_{k m}+a_{m i}+p_{\mathrm{eff}}^{i}-2}-A_{k m i} \eta^{a_{k m}+p_{\mathrm{eff}}^{m}-1}\right] \mathcal{C}_{i}(\Lambda)}{A_{k m} A_{m i} A_{k m i}}$,

where we defined for convenience

$A_{k m}=a_{k m}-p_{\mathrm{eff}}^{k}+p_{\mathrm{eff}}^{m}-1, \quad A_{m i}=a_{m i}+p_{\mathrm{eff}}^{i}-p_{\mathrm{eff}}^{m}-1, \quad A_{k m i}=a_{k m}+a_{m i}+p_{\mathrm{eff}}^{i}-p_{\mathrm{eff}}^{k}-2$.

To compare to the common assumption that $\mathcal{C}_{i}$ and the anomalous dimensions $\gamma_{k m, m i}$ are scale-independent, we observe that these assumptions correspond to the limit

$$
p_{\text {eff }}^{i, m, k} \rightarrow 0, \quad a_{k m, m i} \rightarrow 0
$$

and yield

$$
\mathcal{C}_{k}(\mu)=\frac{p^{k m} p^{m i}}{2}\left[\alpha_{s}(\Lambda)\right]^{-2}\left[1-\eta^{-1}\right]^{2} \mathcal{C}_{i}(\Lambda)
$$

with

$$
p^{k m}=-\frac{\gamma_{k m}}{8 \pi \beta_{0}}, \quad p^{m i}=-\frac{\gamma_{m i}}{8 \pi \beta_{0}} .
$$

Inserting eq. (4.1) one finds

$$
\mathcal{C}_{k}(\mu)=\frac{\gamma_{k m} \gamma_{m i}}{2}\left[\frac{1}{16 \pi^{2}} \ln \left(\frac{\Lambda}{\mu}\right)\right]^{2} C_{i}(\Lambda)
$$

which can also be obtained by integrating the RGEs directly in $\ln \mu$. This serves as a non-trivial crosscheck of eq. (4.24).

\subsection{Generalization to an arbitrary number of operators}

Finally we consider the case with several operators at each level, which is rather common in practical applications. Having shown the effectiveness of our approximation (1.2), we use it in the following for simplicity. As above, we start with the self-mixing of the parent operators $\mathcal{O}_{i}$, denoting $\mathcal{C}_{P}=\left(\mathcal{C}_{i}\right)$ and analogously for child and mediator operators:

$$
\dot{\mathcal{C}}_{P}=\hat{\gamma}^{P} \mathcal{C}_{P}=\alpha_{s} \hat{h}_{\text {eff }}^{P} \mathcal{C}_{P} .
$$

Given the scale-independence of $\hat{h}_{\text {eff }}$ in our approximation, we can easily solve this equation by diagonalization via

$$
\hat{h}_{\mathrm{eff}}^{P}=\hat{V}_{P} \hat{h}_{\mathrm{eff}}^{P, D} \hat{V}_{P}^{-1},
$$

where $\hat{h}_{\text {eff }}^{P, D}$ is a diagonal matrix. Defining furthermore

$$
\mathcal{K}_{P}=\hat{V}_{P}^{-1} \mathcal{C}_{P}
$$

we obtain the equation

$$
\frac{d \mathcal{K}_{P}}{d \alpha_{s}}=\alpha_{s}^{-1} \hat{p}_{\text {eff }}^{P, D} \mathcal{K}_{P}
$$


which decouples the differential equations and has the solution

$$
\begin{array}{rlll}
\mathcal{K}_{P}(\mu)=\hat{U}_{\mathcal{K}}^{P}(\mu, \Lambda) \mathcal{K}_{P}(\Lambda) & \text { with } & \hat{U}_{\mathcal{K}}^{P}(\mu, \Lambda)=\eta^{\mathbf{p}_{\text {eff }}^{P, D}} \equiv \operatorname{diag}\left(\eta^{p_{\text {eff }, j}^{P, D}}\right), \quad \text { or } \\
\mathcal{C}_{P}(\mu)=\hat{U}^{P}(\mu, \Lambda) \mathcal{C}_{P}(\Lambda) \quad \text { with } & \hat{U}^{P}(\mu, \Lambda)=\hat{V}_{P} \hat{U}_{\mathcal{K}}^{P}(\mu, \Lambda) \hat{V}_{P}^{-1},
\end{array}
$$

i.e. the solution is analogous to eq. (4.12), only in the rotated basis. To obtain the solution in practice, one needs to use standard linear algebra methods to obtain $\hat{V}_{P}$ and $\hat{h}_{\mathrm{eff}}^{M, D}$.

To generalize the one-step running, we recall eq. (2.2) and hence consider

$$
\dot{\mathcal{C}}_{C}=\alpha_{s} \hat{h}_{\text {eff }}^{C} \mathcal{C}_{C}+\hat{\gamma}^{P \rightarrow C} \mathcal{C}_{P}, \quad \text { where } \quad\left(\gamma_{k i}^{P \rightarrow C}\right)=\left(\alpha_{s}^{a_{k i}} h_{k i}^{P \rightarrow C}\right)
$$

Diagonalizing the strong mixing matrix for the child operators analogous to eqs. (4.31) and (4.32), and inserting the above solution for $\mathbf{C}_{P}$, we have

$$
\dot{\mathcal{K}}_{C}=\alpha_{s} \hat{h}_{\text {eff }}^{C, D} \mathcal{K}_{C}+\hat{V}_{C}^{-1} \hat{\gamma}^{P \rightarrow C} \hat{V}_{P} U_{\mathcal{K}}^{P}(\mu, \Lambda) \mathcal{K}_{P}(\Lambda) .
$$

This equation is already diagonal in the coefficients $\mathcal{K}_{k}$, but involves now a lengthy sum of terms with explicit $\alpha_{s}$ dependence, which is, however, trivial to treat in the solution of the differential equation: making the $\alpha_{s}$-dependence explicit as before, we have

$$
\begin{aligned}
\frac{d \mathcal{K}_{C}^{k}}{d \alpha_{s}} & =\alpha_{s}^{-1} p_{\mathrm{eff}, k}^{C, D} \mathcal{K}_{C}^{k}+\sum_{i, j, l} B_{k l j i}\left[\alpha_{s}(\Lambda)\right]^{-p_{\mathrm{eff}, i}^{P, D}} \alpha_{s}^{a_{l j}+p_{\mathrm{eff}, i}^{P, D}-2} \mathcal{K}_{P}^{i}(\Lambda), \quad \text { with } \\
B_{k l j i} & =\left(\hat{V}_{C}^{-1}\right)_{k l} p_{l j}^{P \rightarrow C}\left(\hat{V}_{P}\right)_{j i} .
\end{aligned}
$$

This equation can again be solved explicitly:

$$
\mathcal{K}_{C}^{k}(\mu)=\sum_{i, j, l} \frac{B_{k l j i}\left[\alpha_{s}(\Lambda)\right]^{a_{l j}-1}}{a_{l j}+p_{\mathrm{eff}, i}^{P, D}-p_{\mathrm{eff}, k}^{C, D}-1}\left[\eta^{a_{l j}+p_{\mathrm{eff}, i}^{P, D}-1}-\eta^{p_{\mathrm{eff}, k}^{C, D}}\right] \mathcal{K}_{P}^{i}(\Lambda),
$$

which is again clearly the generalization of eq. (4.19) in the (doubly) rotated basis. The solution for the coefficients of child operators in the original basis reads then

$$
\mathcal{C}_{C}(\mu)=\hat{V}_{C} \mathcal{K}_{C}(\mu)
$$

In the limit that the strong operator mixing becomes diagonal for parent and child operators, this equation simplifies to

$$
\mathcal{C}_{C}^{k}(\mu)=\sum_{i} \frac{p_{k i}^{P \rightarrow C}\left[\alpha_{s}(\Lambda)\right]^{a_{k i}-1}}{a_{k i}+p_{\mathrm{eff}, i}^{P}-p_{\mathrm{eff}, k}^{C}-1}\left[\eta^{a_{k i}+p_{\mathrm{eff}, i}^{P}-1}-\eta^{p_{\mathrm{eff}, k}^{C}}\right] \mathcal{C}_{P}^{i}(\Lambda) .
$$

All other effects remain included, and the advantage is that the relevant coefficients can directly be read off the RGEs, without determining the diagonalization matrices. If additionally even the diagonal running of parent or child operators can be neglected, the corresponding $p_{\text {eff }}^{(P / C), D}$ elements vanish.

Finally, we consider two-step mixing with an arbitrary number of operators at each level. This problem is still exactly solvable, but the equations become quite cumbersome, so we defer them to appendix A. However, its solution eq. (A.5) provides an analytic 
master formula that with the help of coefficient tables obtainable from refs. [3-5] allows for the calculation of the two-step evolution down to the electroweak scale, including the relvant running of all anomalous dimensions involved. Furthermore, in all these equations large logarithms are resummed; while this might not be necessary for the weak mixing contributions, it can constitute a large effect for the running due to the top-Yukawa and strong couplings when going to large scales $\Lambda$. For the case of negligible self-mixing the formulae are much simpler: we still consider an arbitrary number of parent operators $\mathcal{O}_{i}$ that generate an arbitrary number of child operators $\mathcal{O}_{k}$ in a two-step running process via an arbitrary number of mediator operators $\mathcal{O}_{m}$, under the assumption that there is no mixing among the operators in each group. Then, simply using eq. (4.24), we find

$$
\mathcal{C}_{k}(\mu)=\sum_{i, m} \frac{p^{k m} p^{m i}}{a_{m i}-1}\left[\alpha_{s}(\Lambda)\right]^{a_{k m}+a_{m i}-2}\left[\frac{\eta^{a_{k m}+a_{m i}-2}-1}{a_{k m}+a_{m i}-2}-\frac{\eta^{a_{k m}-1}-1}{a_{k m}-1}\right] \mathcal{C}_{i}(\Lambda) .
$$

While this equation still looks a bit cumbersome, it has again the advantage that all relevant coefficients can be read off easily, without the need of diagonalization. This is also true for the case of diagonal self-mixing, discussed in appendix A. Yet, one should be aware of the fact that these simpler analytic results do not describe the general case considered in appendix $\mathrm{A}$ in which all parent, mediator and child operators mix with each other. Moreover, generally the generated operators will mix back into their respective parent operators. While the latter effect is suppressed, as discussed above, it is interesting to note that it could in fact be included analytically as well.

\subsection{Special cases of weak mixing}

So far we assumed an arbitrary coefficient for the $\alpha_{s}$-dependence of the weak ADM, parametrized by the $a^{x y}$. Here we discuss a few specific cases in which our formalism yields even simpler expressions.

The trivial case $a_{k i} \rightarrow 0$ is relevant for pure electroweak mixing $\sim g_{1,2}^{2}$ and occurs also when considering the limit of constant ADMs. For the latter limit, we have $p_{\text {eff, } x}^{X} \rightarrow 0$ and $a_{k i} \rightarrow 0$ implying $p_{k i}^{M \rightarrow C} \rightarrow-\gamma_{k i}^{M \rightarrow C} /\left(8 \pi \beta_{0}\right)$, and we obtain from eq. (4.40) the trivial generalization of eq. (3.3).

A less trivial limit is the weak mixing via Yukawa interactions. In this case, the mixing is typically proportional to $y_{q} y_{q^{\prime}}$, and its scale-dependence can be approximated by considering $a_{x y}=1$. Then the general solution for one-step mixing in eq. (4.40) reduces to

$$
\mathcal{K}_{C}^{k}=\sum_{i, j, l} \frac{B_{k l j i}}{p_{\mathrm{eff}, i}^{P, D}-p_{\mathrm{eff}, k}^{C, D}}\left[\eta^{p_{\mathrm{eff}, i}^{P, D}}-\eta^{p_{\mathrm{eff}, k}^{C, D}}\right] \mathcal{K}_{P}^{i}(\Lambda),
$$

with $B_{k l j i}$ given in eq. (4.39). Similarly, the general expression for two-step mixing, given in eq. (A.5), is reduced to

$$
\mathcal{K}_{C}^{k}=\sum_{i, j, l, m, n, o} \frac{E_{k l o m} B_{m n j i}\left[\Delta p_{i m} \eta^{p_{\text {eff }, k}^{C, D}}+\Delta p_{k m} \eta^{p_{\text {eff }, i}^{P, D}}-\Delta p_{i k} \eta^{p_{\text {eff, }, m}^{M, D}}\right] \mathcal{K}_{i}^{P}(\Lambda)}{\Delta p_{i m} \Delta p_{m k} \Delta p_{i k}},
$$

where $E_{k l o m}$ is defined in eq. (A.7) and where we defined

$$
\Delta p_{a b}=p_{\mathrm{eff}, a}^{X_{a}, D}-p_{\mathrm{eff}, b}^{X_{b}, D}, \quad \text { with } \quad X_{a}=\{P, M, C\} \text { for } a \in\{(i, j),(m, n, o),(k, l)\} .
$$




\section{$5 \quad$ Numerical analysis}

In order to facilitate the application of the formalism developed over the last sections, we perform in this section one calculation explicitly. We choose an example where RGE running can be very important, due to the enhancement of certain (left-right) matrix elements for meson mixing [15]. To be even more specific, we consider the case of left-right contributions to $B_{d}$-mixing, and use a basis in which the down-Yukawa matrix is diagonal, implying $Y_{u}=V^{\dagger} Y_{u}^{\text {diag }}$ where $V$ denotes the CKM matrix. In this specific case, only one operator $\mathcal{O}_{H d}$ is created at the NP scale $\Lambda$. Looking up its RGE [4, 5], its self-mixing is approximated via

$$
\left[\dot{\mathcal{C}}_{H d}\right]_{13}=6 y_{t}^{2}\left[\mathcal{C}_{H d}\right]_{13},
$$

from which we can read off, using eqs. (4.30), (4.31) and (4.3), $\hat{V}_{P}=1$ and hence

$$
\hat{h}_{\mathrm{eff}}^{P, D}=\hat{h}_{\mathrm{eff}}^{P}=6 y_{t}\left(\mu_{0}\right)^{2} \frac{\left[\alpha_{s}(\Lambda)\right]^{2 \epsilon_{y_{t}}-1}}{\left[\alpha_{s}\left(\mu_{0}\right)\right]^{2 \epsilon_{y_{t}}}}, \quad \text { and } \quad \hat{p}_{\text {eff }}^{P, D}=\hat{p}_{\mathrm{eff}}^{P}=-\frac{3}{4 \pi \beta_{0}} y_{t}\left(\mu_{0}\right)^{2} \frac{\left[\alpha_{s}(\Lambda)\right]^{2 \epsilon_{y_{t}}-1}}{\left[\alpha_{s}\left(\mu_{0}\right)\right]^{2 \epsilon_{y_{t}}}} .
$$

The child operators consist of $\mathcal{O}_{q d}^{(1,8)}$; the relevant parts of their RGEs read

$$
\begin{aligned}
& {\left[\dot{\mathcal{C}}_{q d}^{(1)}\right]_{1313}=\left(Y_{u} Y_{u}^{\dagger}\right)_{13}\left[\mathcal{C}_{H d}\right]_{13}+\frac{1}{2}\left(Y_{u} Y_{u}^{\dagger}\right)_{33}\left[\mathcal{C}_{q d}^{(1)}\right]_{1313}-\frac{32 \pi}{3} \alpha_{s}\left[\mathcal{C}_{q d}^{(8)}\right]_{1313},} \\
& {\left[\dot{\mathcal{C}}_{q d}^{(8)}\right]_{1313}=\left(\frac{1}{2}\left(Y_{u} Y_{u}^{\dagger}\right)_{33}-56 \pi \alpha_{s}\right)\left[\mathcal{C}_{q d}^{(8)}\right]_{1313}-48 \pi \alpha_{s}\left[\mathcal{C}_{q d}^{(1)}\right]_{1313} .}
\end{aligned}
$$

From these equations we identify $a_{q d(1) H d}=2 \epsilon_{y t}$ together with

$$
\begin{aligned}
\hat{p}_{\text {eff }}^{C} & =-\frac{1}{48 \pi \beta_{0}}\left(\begin{array}{cc}
3\left|V_{t b}\right|^{2} y_{t}^{2} / \alpha_{s} & -64 \pi \\
-288 \pi & 3\left|V_{t b}\right|^{2} y_{t}^{2} / \alpha_{s}-336 \pi
\end{array}\right) \equiv\left(\begin{array}{ll}
x_{11} & x_{12} \\
x_{21} & x_{22}
\end{array}\right) \text { and } \\
\hat{p}^{P \rightarrow C} & =-\frac{1}{8 \pi \beta_{0}}\left(\begin{array}{c}
V_{t d}^{*} V_{t b}\left[y_{t}\left(\mu_{0}\right)\right]^{2}\left[\alpha_{s}\left(\mu_{0}\right)\right]^{-2 \epsilon_{y_{t}}} \\
0
\end{array}\right) .
\end{aligned}
$$

The remaining diagonalization will rarely be done by hand, but for the purpose of this example we give the diagonalization matrix and eigenvalues explicitly: defining

$$
X=\sqrt{\left(x_{11}-x_{22}\right)^{2}+4 x_{12} x_{21}},
$$

we obtain for the eigenvalues

$$
p_{\text {eff }, 1}^{C, D}=\frac{1}{2}\left(x_{11}+x_{22}-X\right) \quad \text { and } \quad p_{\text {eff }, 2}^{C, D}=\frac{1}{2}\left(x_{11}+x_{22}+X\right),
$$

and for the diagonalization matrix

$$
\hat{V}_{C}=\frac{1}{2 x_{21}}\left(\begin{array}{cc}
x_{11}-x_{22}-X & x_{11}-x_{22}+X \\
2 x_{21} & 2 x_{21}
\end{array}\right), \quad \hat{V}_{C}^{-1}=\frac{1}{2 X}\left(\begin{array}{cc}
-2 x_{21} & x_{11}-x_{22}+X \\
2 x_{21} & -x_{11}+x_{22}+X
\end{array}\right) \text {. }
$$

With this, all the ingredients for evaluating eq. (4.40) are given. In figure 4 we show the relative influence of the full running (within our approximations) of parent and children operators, including the mixing among the latter, compared to the trivial running with $\hat{\gamma}_{P \rightarrow C} \equiv$ const., $\hat{\gamma}_{P}=\hat{\gamma}_{C}=0$. We observe a smaller effect on $C_{q d}^{(1)}$ than in the previous example (see figure 3), but still the influence on $C_{q d}^{(1)}$ is $\sim 10 \%$ and $C_{q d}^{(8)}$ is generated at $\sim 25 \%$ of $C_{q d}^{(1)}$ at the electroweak scale when starting from $\Lambda=20 \mathrm{TeV}$. 

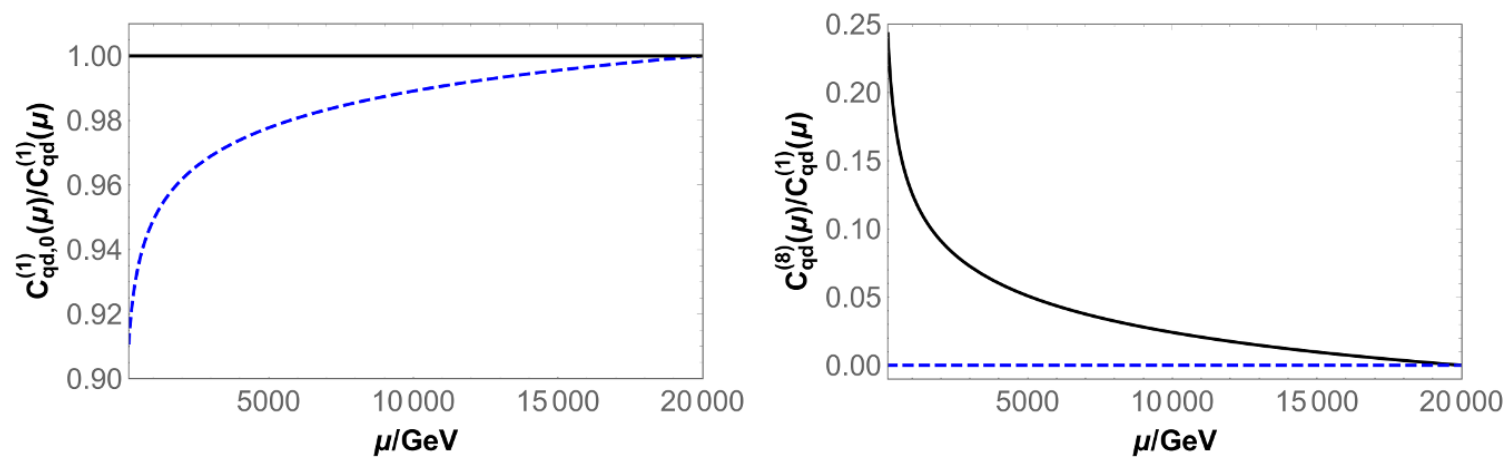

Figure 4. Example of full mixing from $\mathcal{O}_{H d}$ into $\mathcal{O}_{q d}^{(1,8)}$ beween $20 \mathrm{TeV}$ and $\mu_{\mathrm{EW}}$ in our framework, including mixing among parent and child operators, relative to the values generated for $\hat{\gamma}_{P \rightarrow C} \equiv$ const., $\hat{\gamma}_{P}=\hat{\gamma}_{C}=0$ (denoted by $C_{q d, 0}$ and shown blue dashed) considered in [15].

\begin{tabular}{|c|c|c|c|c|c|c|c|c|c|c|}
\hline$P \rightarrow P$ & 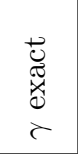 & 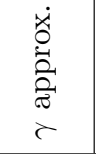 & $\begin{array}{l}+\overrightarrow{0} \\
0 \\
0 \\
0 \\
c\end{array}$ & $P \rightarrow C$ & 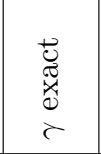 & 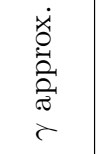 & $\begin{array}{l}\vec{U} \\
00 \\
0 \\
0 \\
c\end{array}$ & $P \rightarrow M \rightarrow C$ & 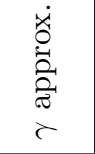 & 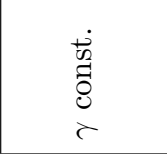 \\
\hline single op. & $(4.9)$ & $(4.12)$ & $(3.1),(3.3)$ & single op. & $(4.15)$ & $(4.19)$ & $(3.1),(3.3)$ & single op. & $(4.24)$ & $(3.1),(3.5)$ \\
\hline multi op. & - & $(4.35)$ & $(3.1),(3.3)$ & multi op. & - & $(4.40)$ & $(3.1),(3.3)$ & multi op. & (A.5) & $(3.1)$ \\
\hline
\end{tabular}

Table 1. Summary of equations relevant for parent-parent mixing $(P \rightarrow P)$, one-loop parent-child mixing $(P \rightarrow C)$ and two-loop parent-mediator-child mixing $(P \rightarrow M \rightarrow C)$ with different levels of approximation.

\section{Summary}

We analyzed the analytic inclusion of significant contributions to the renormalizationgroup evolution from the strong and top-Yukawa couplings to operators generated via weak mixing. This can be achieved due to a distinct hierarchy between several effects, allowing to solve the relevant RGEs in a step-wise fashion. While the running due to both the strong and the top-Yukawa coupling (squared) can be included exactly, the approximation $y_{t}^{2}(\mu) / \alpha_{s}(\mu) \approx$ const. has been shown to be excellent and facilitates the analysis greatly. The included effects are sizable mostly for the coefficients of the generated child operators. The analytic inclusion of these contributions facilitates for instance the application of phenomenlogical constraints at different scales.

Our paper contains a large number of expressions that correspond to various patterns of ADMs and to different approximations. In order to make our paper transparent we summarize the equations corresponding to these different situations in table 1 . In addition to the main formulae listed there, we discuss specific additional approximations in eqs. (4.42)-(4.45) and (A.8) that yield further simplifications.

We hope that the analytic results presented here will give readers better insight into the importance of the various renormalization-group effects than it is possible by using numerical codes, only. Furthermore, the explicit expressions should allow for quick tests of new ideas without getting involved with the intricacies of the codes present in the literature. 


\section{Acknowledgments}

This research was supported by the DFG cluster of excellence "Origin and Structure of the Universe".

\section{A General two-step running with an arbitrary number of operators}

We consider a system of RGEs for two-step running, including strong self-mixing and an arbitrary number of operators at every stage:

$$
\dot{\mathcal{C}}_{P}=\alpha_{s} \hat{h}_{\text {eff }}^{P} \mathcal{C}_{P}, \quad \dot{\mathcal{C}}_{M}=\alpha_{s} \hat{h}_{\text {eff }}^{M} \mathcal{C}_{M}+\hat{\gamma}^{P \rightarrow M} \mathcal{C}_{P}, \quad \dot{\mathcal{C}}_{C}=\alpha_{s} \hat{h}_{\text {eff }}^{C} \mathcal{C}_{C}+\hat{\gamma}^{M \rightarrow C} \mathcal{C}_{M}
$$

where the three matrices $\hat{h}_{\mathrm{eff}}^{X}(X=P, M, C)$ are again approximately scale-independent and defined via

$$
\hat{h}_{\mathrm{eff}}^{X}=\alpha_{s}^{-1} \hat{\gamma}_{X}=\hat{V}_{X} \hat{h}_{\mathrm{eff}}^{X, D} \hat{V}_{X}^{-1} .
$$

The solutions for the self-running of $\mathcal{C}_{P}$ and the full expression for the mediator operators can be obtained from eqs. (4.35) and (4.40). We rewrite the third equation in eq. (A.1) as

$$
\dot{\mathcal{K}}_{C}=\alpha_{s} h_{\text {eff }}^{C, D} \mathcal{K}_{C}+\hat{V}_{C}^{-1} \hat{\gamma}^{M \rightarrow C} \hat{V}_{M} \mathcal{K}_{M}
$$

Inserting the solution for $\mathcal{K}_{M}$, we can further rewrite this equation as

$$
\begin{aligned}
\frac{d \mathcal{K}_{C}^{k}}{d \alpha_{s}}=\alpha_{s}^{-1} p_{\mathrm{eff}, k}^{C, D} \mathcal{K}_{C}^{k}+\sum_{i, j, l, m, n, o} E_{k l o m} \frac{B_{m n j i}}{A_{m n j i}}\left\{\left[\alpha_{s}(\Lambda)\right]^{-p_{\mathrm{eff}, i}^{P, D} \alpha_{s}^{a_{l o}+a_{n j}+p_{\mathrm{eff}, i}^{P, D}-3}-}\right. \\
{\left.\left[\alpha_{s}(\Lambda)\right]^{-p_{\mathrm{eff}, m}^{M, D}+a_{n j}-1} \alpha_{s}^{a_{l o}+p_{\mathrm{eff}, m}^{M, D}-2}\right\} \mathcal{K}_{i}^{P}(\Lambda), }
\end{aligned}
$$

which is solved by

$$
\begin{aligned}
& \mathcal{K}_{C}^{k}= \\
& \sum_{i, j, l, m, n, o} \frac{E_{k l o m} B_{m n j i}\left[\alpha_{s}(\Lambda)\right]^{a_{l o}+a_{n j}-2}\left[A_{m n j i} \eta^{p_{\text {eff }, k}^{C, D}}+A_{k l o m} \eta^{a_{l o}+a_{n j}+p_{\text {eff }, i}^{P, D}-2}-A_{k l o n j i} \eta^{a_{l o}+p_{\text {eff }, m}^{M, D}-1}\right] \mathcal{K}_{i}^{P}(\Lambda)}{A_{m n j i} A_{k l o m} A_{k l o n j i}} .
\end{aligned}
$$

Here we introduced the abbreviations

$$
\begin{aligned}
A_{m n j i} & =a_{n j}+p_{\mathrm{eff}, i}^{P, D}-p_{\mathrm{eff}, m}^{M, D}-1, & & A_{k l o m}=a_{l o}-p_{\mathrm{eff}, k}^{C, D}+p_{\mathrm{eff}, m}^{M, D}-1, \\
A_{k l o n j i} & =a_{l o}+a_{n j}+p_{\mathrm{eff}, i}^{P, D}-p_{\mathrm{eff}, k}^{C, D}-2, & & E_{k l o m}=\left(\hat{V}_{C}^{-1}\right)_{k l} p_{l o}^{M \rightarrow C}\left(\hat{V}_{M}\right)_{o m},
\end{aligned}
$$

and used the definition in eq. (4.39). In the limit where the strong mixing matrices are diagonal, this equation simplifies to

$\mathcal{C}_{C}^{k}=\sum_{i, m} \frac{p_{k m} p_{m i}\left[\alpha_{s}(\Lambda)\right]^{a_{k m}+a_{m i}-2}\left[A_{m m i i} \eta^{p_{\text {eff }, k}^{C}}+A_{k k m m} \eta^{a_{k m}+a_{m i}+p_{\mathrm{eff}, i}^{P}-2}-A_{k k m m i i} \eta^{a_{k m}+p_{\mathrm{eff}, m}^{M}-1}\right] \mathcal{C}_{i}^{P}(\Lambda)}{A_{m m i i} A_{k k m m} A_{k k m m i i}}$,

where the coefficients can be read off directly from the RGEs in [3-5]. 
Open Access. This article is distributed under the terms of the Creative Commons Attribution License (CC-BY 4.0), which permits any use, distribution and reproduction in any medium, provided the original author(s) and source are credited.

\section{References}

[1] W. Buchmüller and D. Wyler, Effective Lagrangian Analysis of New Interactions and Flavor Conservation, Nucl. Phys. B 268 (1986) 621 [INSPIRE].

[2] B. Grzadkowski, M. Iskrzynski, M. Misiak and J. Rosiek, Dimension-Six Terms in the Standard Model Lagrangian, JHEP 10 (2010) 085 [arXiv: 1008.4884] [INSPIRE].

[3] E.E. Jenkins, A.V. Manohar and M. Trott, Renormalization Group Evolution of the Standard Model Dimension Six Operators I: Formalism and lambda Dependence, JHEP 10 (2013) 087 [arXiv: 1308.2627] [INSPIRE].

[4] E.E. Jenkins, A.V. Manohar and M. Trott, Renormalization Group Evolution of the Standard Model Dimension Six Operators II: Yukawa Dependence, JHEP 01 (2014) 035 [arXiv:1310.4838] [INSPIRE].

[5] R. Alonso, E.E. Jenkins, A.V. Manohar and M. Trott, Renormalization Group Evolution of the Standard Model Dimension Six Operators III: Gauge Coupling Dependence and Phenomenology, JHEP 04 (2014) 159 [arXiv: 1312.2014] [INSPIRE].

[6] A. Celis, J. Fuentes-Martin, A. Vicente and J. Virto, DsixTools: The Standard Model Effective Field Theory Toolkit, Eur. Phys. J. C 77 (2017) 405 [arXiv:1704. 04504] [InSPIRE].

[7] J. Aebischer, J. Kumar and D.M. Straub, Wilson: a Python package for the running and matching of Wilson coefficients above and below the electroweak scale, arXiv:1804.05033 [INSPIRE].

[8] The Gambit Flavour Workgroup collaboration, F.U. Bernlochner et al., FlavBit: A GAMBIT module for computing flavour observables and likelihoods, Eur. Phys. J. C 77 (2017) 786 [arXiv: 1705. 07933] [INSPIRE].

[9] J. Hisano, K. Tsumura and M.J.S. Yang, QCD Corrections to Neutron Electric Dipole Moment from Dimension-six Four-Quark Operators, Phys. Lett. B 713 (2012) 473 [arXiv: 1205.2212] [INSPIRE].

[10] J. de Blas, M. Chala and J. Santiago, Renormalization Group Constraints on New Top Interactions from Electroweak Precision Data, JHEP 09 (2015) 189 [arXiv:1507.00757] [INSPIRE].

[11] V. Cirigliano, W. Dekens, J. de Vries and E. Mereghetti, Is there room for CP-violation in the top-Higgs sector?, Phys. Rev. D 94 (2016) 016002 [arXiv:1603.03049] [INSPIRE].

[12] V. Cirigliano, W. Dekens, J. de Vries and E. Mereghetti, Constraining the top-Higgs sector of the Standard Model Effective Field Theory, Phys. Rev. D 94 (2016) 034031 [arXiv: 1605.04311] [INSPIRE].

[13] F. Feruglio, P. Paradisi and A. Pattori, On the Importance of Electroweak Corrections for $B$ Anomalies, JHEP 09 (2017) 061 [arXiv: 1705. 00929] [INSPIRE].

[14] C. Bobeth, A.J. Buras, A. Celis and M. Jung, Patterns of Flavour Violation in Models with Vector-Like Quarks, JHEP 04 (2017) 079 [arXiv: 1609.04783] [INSPIRE]. 
[15] C. Bobeth, A.J. Buras, A. Celis and M. Jung, Yukawa enhancement of Z-mediated new physics in $\Delta S=2$ and $\Delta B=2$ processes, JHEP 07 (2017) 124 [arXiv:1703.04753] [INSPIRE].

[16] K. Fuyuto and M. Ramsey-Musolf, Top Down Electroweak Dipole Operators, Phys. Lett. B 781 (2018) 492 [arXiv: 1706.08548] [INSPIRE].

[17] T.P. Cheng, E. Eichten and L.-F. Li, Higgs Phenomena in Asymptotically Free Gauge Theories, Phys. Rev. D 9 (1974) 2259 [InSPIRE].

[18] M.E. Machacek and M.T. Vaughn, Two Loop Renormalization Group Equations in a General Quantum Field Theory. 2. Yukawa Couplings, Nucl. Phys. B 236 (1984) 221 [inSPIRE].

[19] C. Balzereit, T. Mannel and B. Plumper, The Renormalization group evolution of the CKM matrix, Eur. Phys. J. C 9 (1999) 197 [hep-ph/9810350] [INSPIRE]. 